\title{
O IPAD E OS APLICATIVOS DE JOGOS COMO INSTRUMENTOS COMPLEXOS DE COGNIÇÃO/SUBJETIVAÇÃO EM AUTISTAS
}

\author{
Letícia Staub Limberger ${ }^{1}$ \\ Nize Maria Campos Pellanda ${ }^{2}$
}

\section{RESUMO}

Ao conceber-se esta pesquisa, levou-se em consideração que cada ser humano aprende do seu modo pessoal e único e que os estilos de aprendizagem podem facilitar esse processo. Nesse contexto, o uso de um computador com tela sensível ao toque, com suas características de possibilitar autonomia, interconexões e invenção de caminhos, abre passagem para a potencialização da cognição e subjetivação da criança autista. Sendo assim, esta escrita tem como objetivos investigar as implicações do uso do iPad em crianças autistas para entender como as tecnologias touch podem potencializar a construção do conhecimento e de subjetividade nestes sujeitos. Trata-se de uma pesquisa qualitativa, pois se propôs a trabalhar com os aspectos subjetivos como uma dimensão inseparável de todo o processo cognitivo dos sujeitos. Foram 10 sujeitos de pesquisa, com faixa etária entre 6 e 10 anos, realizando-se 10 sessões com cada sujeito, de forma que em cada encontro foram propostas tarefas desafiadoras a serem realizadas no iPad, levando-se em consideração o diagnóstico de cada sujeito. Foi possível constatar, através dos dados gerados pela pesquisa, que a utilização do iPad pelas crianças que possuem autismo contribuiu em todos os casos estudados de forma positiva para o aprimoramento de questões referentes à sua interação social e aprendizagem.

Palavras-chave: Autismo. Tecnologia. Jogos. iPad. Subjetivação. Cognição.

\begin{abstract}
Throughout this research, we took into consideration that every human being learns in its own personal and unique way, and that learning styles can facilitate this process. In this context, the use of a computer with touchscreen technology, with its characteristics of enabling autonomy, interconnections and invention of paths, open passage to potencialization. Thus, this project aims to investigate the implications of using the $\mathrm{Pad}$ in autistic children to understand how touchscreen technology may enhance the construction of knowledge and subjectivity in these subjects. This is a qualitative research because it proposes to work with the subjective aspects as an inseparable dimension of the cognitive processes of subjects. There were 10 subjects, aged between 6 and 10 years. Ten sessions per subject were performed, so that in each encounter challenging tasks were proposed to be held on the iPad, taking into consideration the diagnosis of each subject. It was possible to see, through the data generated by the research, that use of the iPad for children with autism contributed, in all cases studied, positively to the improvement of issues pertaining to their social interaction and learning.
\end{abstract}

Keywords: Autism. Tchnology. Games. iPad. Subjectivity. Cognition.

\footnotetext{
${ }^{1}$ Mestranda em Educação pela Universidade de Santa Cruz do Sul - UNISC. <lestaub@gmail.com>

${ }^{2}$ Doutora em Educação - Programa de Pós-Graduação - Mestrado Educação - UNISC. <nizepe @ uol.com.br>
} 


\section{INTRODUÇÃO}

Atualmente a tecnologia ocupa um lugar cada vez mais presente em nossa vida, de forma que ultrapassamos a visão ingênua que a coloca como algo que não pode ser incorporado a nossa forma de aprender e viver, e observamos transformações potenciais na vida das pessoas conectadas pela interação. Nesse sentido, buscamos através da pesquisa realizada, trazer a experiência concreta no processo de utilização das tecnologias e, a partir da reflexão sobre a cartografia vivenciada num espaço multimeio, mostrar, como na práxis, pode emergir subjetividade/conhecimento através do uso das tecnologias touch. Esse tipo de tecnologia com tela sensível ao toque dispensa a necessidade de outros periféricos como o teclado, no caso do computador, permitindo um maior acoplamento entre o indivíduo e o aparelho.

Cada ser humano é único e, por isso, aprende do seu modo pessoal e único. Considerando isso, acreditamos que os estilos de aprendizagem podem facilitar esse processo. Um computador com tela sensível ao toque e com características de possibilitar autonomia, interconexões e invenção de caminhos, abre passagem para a potencialização, ou seja, um processo enriquecedor de cognição e subjetivação. O sucesso do iPad com crianças, jovens e idosos vem crescendo rapidamente e, dessa maneira, buscamos investigar qual a ressonância do mesmo com o sistema orgânico/cognitivo/subjetivo humano. Portanto, optou-se por investigar esta interação em sujeitos com diagnóstico autista, visto que a literatura recente tem evidenciado resultados surpreendentes no uso das tecnologias touch por estes sujeitos.

Nosso problema central de pesquisa configurou-se em torno da investigação das implicações do uso do iPad em crianças com patologias graves e leves para entender como as tecnologias touch podem potencializar a construção do conhecimento/subjetividade para estes sujeitos. Dessa forma, buscamos entender o acoplamento sujeito/máquina nas tecnologias touch, observar o processo de complexificação dos sujeitos envolvidos e compreender a cognição de forma ampliada a partir de um artefato técnico.

Acreditamos ser de suma importância esclarecer que através desse artigo não pretendemos pôr um ponto final no assunto, visto que estudos sobre o iPad são muito recentes e raros, mas que nossa intenção é cartografar alguns elementos para dar contribuições nesse caminho que começa a ser trilhado. Dessa forma, nossas metas configuraram-se em gerar subsídios para melhorar o atendimento clínico e didático de crianças com patologias e para que, em cima desses dados, se possa desenvolver maneiras de utilizar os resultados de forma mais ampla em todas as crianças da escola fundamental. Portanto, a escrita desse artigo serve como arcabouço teórico para pensarmos as possibilidades de desenvolvimento de aplicativos 
a partir dos referenciais teóricos da segunda cibernética, ou seja, os saberes reflexos do aprender a aprender e outros que aportem maior autonomia aos sujeitos.

\section{FUNDAMENTAÇÃO TEÓRICA}

Buscamos entender melhor os dados gerados pela pesquisa através de uma estrutura teórica que nos serviu de suporte, na qual contamos com os estudos originários na Segunda Cibernética de Von Foerster (2003) e seus desdobramentos na Biologia da Cognição de Humberto Maturana e Francisco Varela (1980) e da Complexificação pelo Ruído de Henri Atlan (1992).

Através da leitura desses e de outros autores citados nesse artigo, buscamos fazer um entendimento acerca de como a cognição dos seres humanos possui uma relação com o ambiente (MATURANA, 1984) - onde o mesmo funciona em termos de circularidade produtiva - e de que maneira o $i P a d$ e os jogos que nele se encontram apresentam-se ao indivíduo autista como um dos recursos desejáveis do seu ambiente, capaz de mudanças e melhora no diagnóstico.

\subsection{Autismo}

O autismo enquanto expressão foi usada pela primeira vez por Bleurer em 1911, sendo que o mesmo buscava, através desse termo, explicitar a perda de contato com a realidade, que por consequência gerava uma grande dificuldade de comunicação. Em 1943, Kanner, em estudos realizados com crianças, utilizou novamente a expressão para descrever certa inabilidade inata para estabelecer contato interpessoal e afetivo que apresentavam os sujeitos de sua pesquisa.

De acordo com Rutter e Schopler (1978) o autismo pode ser considerado um distúrbio de desenvolvimento complexo, com características acentuadas no campo do comportamento e com etiologias múltiplas e graus variados de severidade. É uma síndrome da família dos transtornos de neurodesenvolvimento, nos quais ocorre uma ruptura nos processos fundamentais de socialização, comunicação e aprendizagem, coletivamente conhecidos como transtornos invasivos de desenvolvimento.

O autismo possui um início precoce (0 a 3 anos de idade), perfil sintomático e cronicidade que envolvem mecanismos biológicos fundamentais relacionados à adaptação social. Existe um marcado e permanente prejuízo na interação social, alterações da comunicação e padrões limitados ou estereotipados de comportamentos e interesses. De 
acordo com a American Psychiatric Association (2002), atrasos na linguagem comunicativa, interação social e comportamentos estereotipados devem estar presentes até os 3 anos de idade.

Grinker (2010) postula que o autismo é um distúrbio cerebral que afeta pessoas em qualquer cultura, entretanto ressalta que, mesmo se tratando de uma doença biológica, ela não pode ser definida única e puramente pela biologia de laboratório. Enfatiza a importância do meio, de como a sociedade em que se encontra o indivíduo com autismo, vê a doença.

muitas sociedades, por exemplo, sequer possuem uma palavra para denominar o autismo, enquanto em outras os sintomas do autismo não são considerados anômalos, ou são vistos como divinos e espirituais (p. 13).

Levando em consideração que a sociedade em que vivemos pertence a uma era digital, nos pareceu pertinente pesquisar como se dá essa interação da criança autista com o meio digital, e de que forma essa nova relação pode contribuir para uma melhor qualidade de vida da criança e, futuramente, adulto.

\subsection{Teconologias}

Para o desenvolvimento da pesquisa com os sujeitos utilizou-se o iPad, que se configura por ser um dispositivo em formato tablet produzido pela Apple. Esse dispositivo se utiliza do sistema operacional $i O S$ e dentre suas especificações técnicas estão incluídos wi-fi, tela de 9,7 polegadas sensível ao toque, acelerômetro, bússola, entre outros. O aparelho possibilita variadas formas de uso, sendo elas mídia áudiovisual caracterizada por livros, filmes, música, jogos e aplicativos variados a serem escolhidos pelos usuários. Observou-se uma grande procura por parte das crianças pelos aplicativos de jogos disponíveis no iPad, tanto nas sessões com as terapeutas quanto em casa. Suas preferências estavam voltadas para estes, de forma que exploraremos um pouco mais sua temática e como se fazem presentes na nossa sociedade.

A realidade social do homem moderno sempre possuiu elementos de jogos nela inseridos. Vários dos passatempos mais populares do nosso tempo, como o xadrez, jogos de cartas e esportes têm raízes que datam de centenas ou mesmo milhares de anos atrás (HUIZINGA, 1950). O ato de jogar é um comportamento que pode ser identificado tanto em animais como em seres humanos. Ao estudar o tema, Vieira e Sartório (2002) concluíram que os jogos podem ser entendidos como um meio para a compreensão do desenvolvimento comportamental e social. Este aspecto tem grande importância para a pesquisa que vem sendo desenvolvida, visto que a grande dificuldade dos autistas está em seu comportamento social e 
cognitivo.

A preferência dos sujeitos dessa pesquisa pelos jogos disponíveis no iPad não passou despercebida e uma das conjecturas que nós enquanto pesquisadores podemos nos apoiar para pensar o motivo por trás dessa escolha é bem explanado por Pereira, Neca, Facchini, Lima e Freitas (2012), os quais ressaltam que:

os videogames tratam de aventuras com protagonistas que exploram ambientes
desconhecidos, desvelam tramas, estabelecem relações e crescem à medida que o
jogo progride. Retratar tais aspectos permeando-os de emoções humanas é nos dias
de hoje uma preocupação de criadores de jogos tão relevante quanto a do
desenvolvimento tecnologico (p. 82).

Nesse sentido, acreditamos que as crianças se projetam enquanto os protagonistas da trama que exploram nos ambientes virtuais e, em especial nessa pesquisa que trabalha com sujeitos autistas, podem estar buscando através do jogo explorar esse aspecto permeado de emoções que este proporciona sem que haja uma real situação de relação com o outro. Nesse sentido, poderíamos pensar por dois ângulos: aquele que acredita que essa situação só tende a intensificar a situação do autista, colocando-o cada vez mais fundo dentro de sua concha; ou, aquele que decidimos ser o mais provável no qual o jogo age como um simulador de realidade, onde há uma diminuição da ansiedade, que permite à criança entender melhor como funcionam as relações e começar a obter maior sucesso quando elas se tornam concretas. Guattari possui um pensamento semelhante:

O que importa aqui não é unicamente o confronto com uma nova matéria de expressão, é a constituição de complexos de subjetivação: indivíduo-grupomáquina-trocas múltiplas que oferecem à pessoa possibilidades diversificadas de recompor uma corporeidade existencial, de sair de seus impasses repetitivos e, de alguma forma, de se re-singularizar (GUATTARI, 1992, p. 17).

Teoricamente, para a construção desse artigo, buscamos sustentação ao concebermos que a tecnologia está integrada à experiência humana desde nossa constituição através da linguagem. Goody (2007) aponta duas hipóteses:

\footnotetext{
a de que o humano surge com a linguagem e a de que o modo de comunicação verbal implica no surgimento de uma tecnologia. Então, desde o princípio, tudo o que fazemos ocorre no acoplamento com tecnologias com que fomos inventando modos de viver e de conhecer (p. 193).
}

Turkle (1997) foca suas pesquisas na relação sujeito/computador, apontando que sua atração pelos computadores está relacionada às possibilidades de conversar que estes oferecem. Para Turkle, este conversar se encontrava em um sistema no qual sujeitos e máquinas formavam um todo complexo e os sujeitos se transformavam, pois o computador era um objeto com o qual pensar. Aponta, dessa forma, que as relações entre seres humanos e 
computadores estão se tornando mais intensas, o que tende a modificar a maneira como elas pensam e sentem.

Seguindo essa linha de raciocínio, Simondon (1989) posiciona-se quanto ao objeto técnico (OT) de maneira complexa ao pensar a técnica na sua dimensão epistêmica e ontológica de forma inseparável. Nesse sentido, propõe-se que a relação dos sujeitos com o artefato tecnológico, no nosso caso o iPad, é uma relação constitutiva em termos cognitivos, sendo estes entendidos neste artigo como inseparáveis de tudo que constitui o humano. Portanto, estamos pensando estes artefatos tecnológicos enquanto possibilidades para construção de significados no sentido de ampliação dos potenciais humanos de conhecimento e invenção de formas de viver.Kastrup (1999) possui ideias semelhantes quando postula:

\footnotetext{
A técnica não é somente o terreno dos objetos artificiais, mas potência de artificialização da cognição e de virtualização da inteligência. Não artificializa uma natureza dada, mas reverbera sobre a natureza da cognição, natureza em si mesma artificiosa e inventiva, que a vida virtual prepara. Abre-se assim a possibilidade de pensar a cognição como híbrido de natureza e artifício (p.183).
}

Portanto, se nos apegarmos ao trecho que reflete que a vida virtual prepar para a realidade, como salientou Kastrup (1999), podemos continuar nossas reflexões observando como muitos dos aplicativos de jogos escolhidos pelas crianças desse estudo têm como função simular situações possíveis, alguns mais diretos, outros mais fantasiosos, mas sempre simulações que preparam para o real. Alguns jogos simulam a hora do banho, de escovar os dentes, de cuidar de animais de estimação, outros tornam-se mais fantasiosos e simulam como fugir de um monstro, como escapar de um labirinto encantado e como salvar a princesa. Sempre há a manutenção de elementos reais em suas fantasias, que fazem com que as crianças se reconheçam em certo nível enquanto personagem principal e busquem formas de ultrapassar as barreiras impostas pelos jogos.

Baum e Maraschin (2011), no que se refere aos jogos eletrônicos, postulam que a solução de problemas não é a única finalidade dos jogos. Eles continuam sua ação na cognição do sujeito através da problematização, ou seja, "a cognição problematiza a si mesma, uma vez que faz parte de sua operação diferenciar-se, problematizar os limites dentro dos quais opera" (p. 42). Ao assumir essa postura entendemos que os jogos proporcionam uma experiência que permite ao sujeito atualizar-se frente a sua própria existência.

Se levarmos em consideração que as crianças desse estudo possuem problemas cognitivos e de comunicação, se torna claro que a possibilidade de vivenciar certas dificuldades primeiramente através de um artefato digital pode significar uma grande diminuição na sua ansiedade frente às adversidades do mundo. Há, também, a geração da 
possibilidade de experienciar mecanismos de autorregulação, que posteriormente serão incorporados pelos sujeitos, tornando-os capazes de autorregular-se.

\section{MATERIAIS E MÉTODOS}

Trata-se de uma pesquisa qualitativa, visto que se propôs a trabalhar com aspectos subjetivos como uma dimensão inseparável de todo o processo cognitivo dos sujeitos. O que foi observado se referiu aos aspectos de emergência de transformações cognitivas e afetivas destas crianças. Os sujeitos da pesquisa foram 10 crianças distribuídas da seguinte maneira: 2 sujeitos diagnosticados como autistas numa primeira etapa do projeto (funcionou como projeto-piloto) atendidas na sala de espelhos do SIS (Sistema Integrado de Saúde) da UNISC; 4 sujeitos na sequência a também atendidos na sala de espelhos do SIS; 4 crianças autistas atendidas na Prefeitura da cidade de Vera Cruz por psicopedagogas e psicólogas locais e supervisionadas pela equipe envolvida na pesquisa.

Após definidos os sujeitos, a pesquisa teve sua continuidade dada através de 10 sessões com cada sujeito, sendo que em cada um desses encontros foram propostas tarefas desafiadoras a serem realizadas no $i P a d$, levando-se em consideração o diagnóstico de cada sujeito. Estas tarefas estão em consonância com os pressupostos teóricos do projeto em termos de desafios para proporcionar atitudes de auto-organização. No final de cada encontro houve uma conversa com as crianças sobre como se sentiram com a realização das tarefas na medida em que o diagnóstico permite a comunicação desses sujeitos. Houve também momentos de conversa com os pais, nos quais os pesquisadores buscavam averiguar as mudanças que estes haviam percebido em seus filhos em casa. Os pesquisadores fizeram um diário de bordo, no qual se incluíram como parte da realidade observada e relataram o processo de acoplamento tecnológico das crianças com o iPad com vistas à questão da complexificação. Foram feitas também filmagens das sessões e reuniões para discussão dos dados.

O tratamento dos dados deu-se coerentemente com nossa postura epistemológica complexa de abordagem da realidade como fluxos e devires. Não usamos categorias fixas de análise, mas sim marcadores teóricos oriundos de nosso quadro teórico para sinalizar passagens significativas no processo de cada criança. Dessa forma, foram usados os seguintes marcadores: construção de autopoiesis, acoplamento tecnológico, e processo de complexificação a partir do ruído. Às conversas com os sujeitos e aos diários de bordo dos pesquisadores foram aplicados os mesmos marcadores. Dessa forma, ao final, o trabalho 
interpretativo com as diferentes fontes de dados gerados foi tramado com vistas a responder ao problema central de pesquisa.

\section{RESULTADOS}

Houve uma melhora gradual e constante nos sintomas de autismo apresentados pelas crianças desse estudo. Devemos ressaltar que pelo fato de o autismo ser um transtorno muito amplo e possuir vários níveis dentro de sua descrição, a melhora de cada sujeito difere do seguinte. Entretanto, não podemos negligenciar as vitórias diárias enfrentadas pelas crianças, suas famílias e pesquisadores, visto que de acordo com os relatos semanais dos pais, assim como interações e observações feitas pelos pesquisadores, foi possível acompanhar o crescimento cognitivo, social e emocional apresentado pelas crianças.

Nossos resultados positivos nesse estudo somente deram início ao trabalho proposto por esta pesquisa, visto que a partir dos dados gerados os pesquisadores foram em busca de respostas e entendimentos para resultados tão satisfatórios. Nesse sentido, algumas de nossas conjecturas giram em torno do conceito de autopoiesis, que se refere a uma dinâmica circular autoprodutiva dos organismos vivos. Ao partimos desse conceito para pensar os resultados dessa pesquisa, podemos entender o iPad e suas possibilidades como uma ferramenta encontrada no meio por esses sujeitos, o que permitiu uma maior congruência desse meio, saindo de um processo que pode ser considerado destrutivo para um processo produtivo.

Podemos agora avançar para a definição de aprendizagem, ao nível biológico, considerando que ela se refere ao processo de transformação do comportamento de um organismo; esse processo de transformação ocorre pela experiência vivencial de cada ser vivo e encontra-se sempre subordinado à conservação da sua lógica organizacional, de um modo direto ou indireto (OLIVEIRA, 1999 pg. 38).

A plasticidade cerebral é potencializada pelas experiências vividas, ou seja, ao resistirmos às mudanças limitaremos novas possibilidades de estímulos ambientais. Rotta postula que:

As mudanças ambientais interferem na plasticidade cerebral e, consequentemente, na aprendizagem. Definida a aprendizagem como modificações do SNC, mais ou menos permanentes, quando o indivíduo é submetido a estímulos e/ou experiências de vida, que vão traduzir em modificações cerebrais. Dessa forma fica bem claro que as alterações plásticas são as formas pelas quais se aprende (ROTTA, 2006, pg. 453). 


\section{CONCLUSÕES}

Através dessa escrita ficou claro que o autismo é um terreno fértil para investigação, visto que ainda existem tantas perguntas a seu respeito não respondidas. Apesar de não termos todas as respostas enquanto cientistas, nos contentamos com algumas conjecturas e com os resultados positivos encontrados através dessa caminhada.

Recentemente, para além dos estudos sobre o universo das tecnologias, a questão dos dispositivos tecnológicos vem recebendo um olhar mais atento na academia quanto às infinitas possibilidades de utilização no setor educacional. Portanto, esperamos que o presente artigo gere contribuições para o desenvolvimento de novas possibilidades de acoplamentos e para a construção de novas formas de aprendizagem. Esperamos também que, do ponto de vista teórico, possamos fomentar cada vez mais discussões que levem à potencialização da subjetividade dos sujeitos usuários das tecnologias touch, seja qual for sua dificuldade.

Enfim, estudos nessa área se mostram sempre importantes, visto que eles proporcionam grandes avanços no que se refere a uma patologia sem cura. Devemos almejar sempre os melhores resultados, entretanto, enquanto pesquisadores, fica claro que a ciência se constrói a passos curtos. Esse foi o primeiro passo na direção de uma vida mais emancipada para essas crianças e muitos outros ainda deverão seguir.

\section{REFERÊNCIAS}

AMERICAN PSYCHIATRIC ASSOCIATION. Manual diagnóstico e estatístico de transtornos mentais: DSM-IV. 4 ed. Porto Alegre: Artes Médicas, 2002.

ATLAN, H. Entre o cristal e a fumaça. Rio de Janeiro: Zahar, 1992.

GOODY, J. Pouvoirs et savoirs de l'écrit. Paris: Editions La Dispute, 2007.

GRINKER, R. R. Autismo: um mundo obscuro e conturbado. São Paulo: Larrousse do Brasil, 2010.

GUATTARI, F. Caosmose. São Paulo: Editora 34, 1992.

HUIZINGA, J. Homo Ludens: a Study of the Play-Element in Culture. The Beacon Press: Boston, 1950.

KASTRUP, V. A invenção de si e do mundo. Campinas: Papirus, 1999.

MATURANA, H. R. Fenomenología del conocer. Santiago: Editorial Universitaria, 1984.

MATURANA, H.; VARELA, F. Biology anf Cognition. Boston: Reidl, 1980. 
OLIVEIRA, C. C. A educação como processo auto-organizativo. Coimbra: Horizontes Pedagógicos, 1999.

PEREIRA, O. C. N.; NECA, L. O.; FACCHINI, A. H.; LIMA, T. P.; FREITAS, L. V. Jogar Videogame como uma Experiência Simbólica: Entrevistas com Jogadores. Boletim de Psicologia, São Paulo, v. 62, n. 136, p. 81-91, 2012.

ROTTA, N. T.; OHLWEILER, L; RIESGO, R. S. Transtornos de aprendizagem: abordagem neurobiológica e multidisciplinar. Porto Alegre: Artmed, 2006.

RUTTER, M.; SCHOPLER, E. Language disorder and infantile autism. Plenum, New York, v. 27, n. 6, p. 85-104, 1978.

SIMONDON, G. Du mode d'existence des objets techniques. Paris: Aubier Philosophi, 1989.

TURKEL, S. Life on the Screen. New York: Touchstone, 1997.

Von FOERSTER, H. Understanding, understanding. New York: Spring, 2003.

VIEIRA, M. L.; SARTÓRIO, R. Análise motivacional, causal e funcional da brincadeira em duas espécies de roedores. Estudos de Psicologia, Natal, v. 7, n. 1, p. 189-196, 2002. 